\title{
CCD and micrometric observations of visual double stars
}

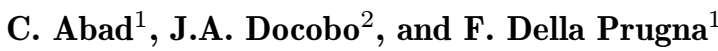 \\ 1 Centro de Investigaciones de Astronomía CIDA, 5101-A Mérida, Venezuela \\ 2 Observatorio Astronómico Ramón María Aller, Universidade de Santiago de Compostela, 15076, Santiago de Compostela, \\ España
}

Received February 12; accepted May 15, 1998

\begin{abstract}
Relative positions and separations for 65 visual binaries are given. CCD frames were taken at the 1-m coudé telescope and micrometric observations were carried out at the $65-\mathrm{cm}$ refractor, both at the Venezuelan National Observatory. The visual double stars observed were selected from the Ramón María Aller Observatory program list. In addition, we also observed several long period double stars with poorly determined orbital elements.
\end{abstract}

Key words: astrometry — binaries: visual

\section{Introduction}

In 1994, a scientific collaboration between the Centro de Investigaciones de Astronomía (CIDA, Venezuela) and the Ramón María Aller Observatory (University of Santiago de Compostela, Spain) began with the purpose to carry out joint research on double and multiple stars, especially to gather observational data for southern stars from the Venezuelan National Observatory. As a result of this program, we report here relative positions of several binaries.

\section{Observations}

Observations were carried out from May 1994 to August 1997 at the Venezuelan National Observatory. Two telescopes were used: the 1-m coudé reflector, equipped with a CCD camera, and the $65-\mathrm{cm}$. Zeiss refractor with a filar micrometer. The CCD camera is based on a Thomson TH-7883 chip, which features $574 \times 384$ pixels $23 \mu \mathrm{m}$ square, giving a scale factor of $0.23 \mathrm{arcsec} / \mathrm{pixel}$ at the coudé focus. However, when the seeing conditions were good, a $1.5 \times$ Barlow lens was used sparingly. The technique adopted to take CCD frame has been described in

Send offprint requests to: C. Abad a previous paper (Abad \& Della Prugna 1994). In brief, multiple images of the visual binary are taken onto a single CCD frame, shifting the telescope in R.A. by a small amount between exposures. To determine the scale factor, double stars from the Second List of Wide Visual Binaries were observed (Brosche \& Sinachopoulos 1989). These were also observed at the refractor, in order to ascertain the screw-value of the filar micrometer. Previous micrometric observations at this telescope were carried out by A. Valbousquet (Valbousquet 1980) and C. Prieto (Prieto 1997). In this work, micrometric observations were carried out by one of the authors (FDP).

\section{Results}

The following table gives relevant data for the visual binaries observed. An asterisk indicates a micrometric observation. All CCD measurements include results from each observation. When several measurements with similar epochs are available for the same double star, the mean Separation and Position Angle are given for the mean epoch after the dashed line. Errors are calculated from the totality of images of the pair for similar epochs. Stars in the table are arranged in increasing R.A. and the magnitudes of the pair are those given in the original list. The table is arranged in the following order:
WDS
WDS catalogue number
(Worley \& Douglass 1996),
ADS or Name ADS number or name of the pair
(Aitken R.G. 1932),
magnitudes magnitudes of the components
(Worley \& Douglass 1996),
epoch of the observations,
$t$
Position Angle in degrees, error of Position Angle,
number of observations for Position Angle.
Separation of the components in arcsecs, error of Separation, number of observations for Separation, 
Table 1. Data for the visual binaries observed

\begin{tabular}{|c|c|c|c|c|c|c|c|c|c|}
\hline$W D S$ & ADS or NAME & magnitudes & $t$ & $\theta$ & $\sigma_{\theta}$ & $n_{\theta}^{0}$ & $\rho$ & $\sigma_{\rho}$ & $n_{\rho}^{0}$ \\
\hline \multirow[t]{6}{*}{$00184+4401$} & $246 A B$ & $8.1-11.0$ & 1997.660 & 63.69 & 0.09 & 3 & 35.30 & 0.04 & 3 \\
\hline & & & 1997.660 & 64.18 & 0.04 & 3 & 35.25 & 0.02 & 3 \\
\hline & & & 1997.663 & 63.92 & 0.04 & 3 & 35.19 & 0.05 & 3 \\
\hline & & & 1997.663 & 64.38 & 0.04 & 3 & 35.24 & 0.06 & 3 \\
\hline & & & ---- & ---- & ---- & -- & ---- & -- & -- \\
\hline & & & 1997.662 & 64.04 & 0.27 & 11 & 35.24 & 0.06 & 11 \\
\hline \multirow[t]{8}{*}{$01398-5612$} & $D U N 5$ & $5.9-5.8$ & 1994.948 & 191.36 & 0.51 & 5 & 10.96 & 0.05 & 3 \\
\hline & & & 1995.025 & 192.28 & 0.25 & 8 & 11.27 & 0.05 & 6 \\
\hline & & & 1997.660 & 191.00 & 0.21 & 5 & 11.35 & 0.04 & 5 \\
\hline & & & 1997.660 & 192.10 & 0.22 & 6 & 11.25 & 0.08 & 6 \\
\hline & & & 1997.666 & 191.71 & 0.33 & 4 & 11.26 & 0.06 & 3 \\
\hline & & & 1997.666 & 191.85 & 0.15 & 4 & 11.24 & 0.05 & 3 \\
\hline & & & ---- & ---- & ---- & -- & ---- & -- & -- \\
\hline & & & 1996.702 & 191.73 & 0.43 & 29 & 11.28 & 0.08 & 29 \\
\hline \multirow[t]{5}{*}{$01456-2503$} & $1394 A B$ & $5.5-8.3$ & 1994.866 & 26.68 & 0.14 & 8 & 4.95 & 0.01 & 8 \\
\hline & & & 1994.874 & 26.76 & 0.14 & 8 & 4.96 & 0.01 & 8 \\
\hline & & & 1994.874 & 27.21 & 0.16 & 6 & 4.92 & 0.02 & 5 \\
\hline & & & ---- & ---- & ---- & -- & ---- & -- & -- \\
\hline & & & 1994.871 & 26.86 & 0.26 & 21 & 4.94 & 0.02 & 21 \\
\hline $01456-2503$ & $1394 A B$ & $5.5-8.3$ & 1997.663 & 25.89 & 0.60 & 4 & 4.87 & 0.03 & 3 \\
\hline $02020+0246$ & $1615 A B$ & $4.3-5.2$ & 1997.663 & 273.88 & 0.51 & 3 & 1.74 & 0.04 & 3 \\
\hline \multirow[t]{4}{*}{$02198-3527$} & HJ 3494 & $8.8-9.0$ & 1994.874 & 253.69 & 0.48 & 6 & 2.03 & 0.01 & 6 \\
\hline & & & 1995.025 & 250.39 & 0.86 & 4 & 1.97 & 0.03 & 4 \\
\hline & & & ---- & ---- & ---- & -- & ---- & -- & -- \\
\hline & & & 1994.934 & 252.37 & 1.74 & 10 & 2.00 & 0.03 & 10 \\
\hline $02442+4914$ & $2081 A B$ & $4.1-10.0$ & 1994.866 & 304.01 & 0.14 & 6 & 20.07 & 0.07 & 6 \\
\hline $02475+1922$ & $2122 A B$ & $7.4-8.3$ & 1994.866 & 308.87 & 0.07 & 6 & 3.63 & 0.01 & 6 \\
\hline \multirow[t]{5}{*}{$03121-2859$} & 2402 & $4.0-6.6$ & 1994.863 & 299.98 & 0.26 & 8 & 4.56 & 0.03 & 8 \\
\hline & & & 1994.874 & 299.26 & 0.30 & 7 & 4.56 & 0.03 & 7 \\
\hline & & & 1995.025 & 299.34 & 0.87 & 4 & 4.57 & 0.09 & 4 \\
\hline & & & ---- & ---- & ---- & -- & ---- & -- & -- \\
\hline & & & 1994.901 & 299.58 & 0.58 & 19 & 4.56 & 0.05 & 19 \\
\hline \multirow[t]{4}{*}{$03122+3713$} & 2390 & $8.1-8.3$ & 1994.863 & 126.84 & 0.45 & 4 & 2.71 & 0.01 & 4 \\
\hline & & & 1994.863 & 127.26 & 0.09 & 6 & 2.71 & 0.01 & 6 \\
\hline & & & ---- & ---- & ---- & -- & ---- & -- & -- \\
\hline & & & 1994.863 & 127.20 & 0.15 & 10 & 2.71 & 0.01 & 10 \\
\hline
\end{tabular}


Table 1. continued

\begin{tabular}{|c|c|c|c|c|c|c|c|c|}
\hline$W D S$ & ADS or NAME & magnitudes & $t$ & $\theta$ & $\sigma_{\theta}$ & $n_{\theta}^{0}$ & $\rho$ & $\sigma_{\rho}$ \\
\hline \multirow{4}{*}{$03368+0035$} & 2644 & $5.9-8.8$ & 1994.866 & 260.23 & 0.28 & 5 & 6.63 & 0.03 \\
\hline & & & 1995.025 & 269.06 & 1.00 & 4 & 6.64 & 0.04 \\
\hline & & & ---- & ---- & ---- & -- & ---- & -- \\
\hline & & & 1994.946 & 269.15 & 0.70 & 9 & 6.63 & 0.03 \\
\hline $03485-3147$ & HJ3596 & $8.3-8.6$ & 1994.948 & 137.92 & 0.30 & 6 & 9.03 & 0.03 \\
\hline \multirow[t]{5}{*}{$03522-4133$} & $R S T 1260$ & $10.9-11.1$ & 1994.866 & 285.81 & 1.04 & 5 & 2.14 & 0.05 \\
\hline & & & 1994.874 & 286.17 & 0.24 & 4 & 2.13 & 0.02 \\
\hline & & & 1995.025 & 287.88 & 1.86 & 5 & 2.12 & 0.03 \\
\hline & & & ---- & ---- & ---- & -- & ---- & -- \\
\hline & & & 1994.925 & 286.37 & 1.24 & 14 & 2.13 & 0.04 \\
\hline \multirow[t]{4}{*}{$03575-0101$} & 2894 & $8.2-10.2$ & 1994.863 & 16.70 & 0.21 & 6 & 11.04 & 0.02 \\
\hline & & & 1994.866 & 17.26 & 0.20 & 7 & 11.07 & 0.03 \\
\hline & & & ---- & ---- & ---- & -- & ---- & -- \\
\hline & & & 1994.865 & 17.00 & 0.35 & 13 & 11.06 & 0.03 \\
\hline $04037-4745$ & $H U 1362$ & $9.5-10.5$ & 1994.866 & 56.38 & 0.31 & 5 & 2.44 & 0.04 \\
\hline $04153-0739$ & $3093 B C$ & $9.5-11.2$ & 1995.025 & 336.82 & 0.16 & 5 & 8.89 & 0.02 \\
\hline $04166-1005$ & $B U 548$ & $7.5-12.0$ & 1994.948 & 344.58 & 0.42 & 3 & 6.26 & 0.03 \\
\hline $04403-5857$ & HJ3683 & $7.3-7.5$ & 1995.025 & 91.77 & 0.21 & 5 & 3.25 & 0.02 \\
\hline $04496-5353$ & $I 342$ & $8.4-8.8$ & 1995.025 & 141.31 & 0.81 & 7 & 2.82 & 0.01 \\
\hline \multirow[t]{5}{*}{$05110+0245$} & $H E I 652$ & $9.8-10.1$ & 1994.863 & 54.67 & 1.34 & 6 & 1.63 & 0.04 \\
\hline & & & 1994.874 & 54.43 & 0.17 & 3 & 1.60 & 0.02 \\
\hline & & & 1995.027 & 55.64 & 1.47 & 5 & 1.60 & 0.01 \\
\hline & & & ---- & ---- & ---- & -- & ---- & -- \\
\hline & & & 1994.920 & 54.97 & 1.34 & 16 & 1.61 & 0.02 \\
\hline \multirow[t]{4}{*}{$05258-2721$} & $R S T 133$ & $10.6-10.6$ & 1994.874 & 164.21 & 0.33 & 4 & 1.31 & 0.03 \\
\hline & & & 1994.874 & 163.20 & 1.15 & 3 & 1.28 & 0.01 \\
\hline & & & ---- & ---- & ---- & -- & ---- & -- \\
\hline & & & 1994.874 & 164.12 & 0.49 & 8 & 1.30 & 0.03 \\
\hline (*)05308+0557 & 4115 & $4.5-5.7$ & 1995.100 & 48.30 & 0.40 & 2 & 1.22 & 0.08 \\
\hline \multirow[t]{5}{*}{$05364+2200$} & 4200 & $7.2-7.8$ & 1994.863 & 272.54 & 0.05 & 7 & 3.99 & 0.01 \\
\hline & & & 1995.025 & 272.65 & 0.23 & 4 & 3.98 & 0.02 \\
\hline & & & 1995.112 & 272.74 & 0.18 & 4 & 3.96 & 0.02 \\
\hline & & & ---- & ---- & ---- & -- & ---- & -- \\
\hline & & & 1994.973 & 272.62 & 0.18 & 15 & 3.98 & 0.02 \\
\hline$(*)$ & 4200 & $7.2-7.8$ & 1995.100 & 272.20 & 0.50 & 2 & 4.03 & 0.09 \\
\hline
\end{tabular}


Table 1. continued

\begin{tabular}{|c|c|c|c|c|c|c|c|c|}
\hline$W D S$ & ADS or NAME & magnitudes & $t$ & $\theta$ & $\sigma_{\theta}$ & $n_{\theta}^{0}$ & $\rho$ & $\sigma_{\rho}$ \\
\hline $05408+0329$ & HEI666 & $9.8-11.9$ & 1994.863 & 249.87 & 1.03 & 3 & 2.43 & 0.03 \\
\hline$(*) 05407-0157$ & $4263 A B$ & $2.0-4.2$ & 1994.500 & 164.50 & 0.80 & 2 & 2.33 & 0.11 \\
\hline $05467-2328$ & $R S T 2397$ & $10.0-10.0$ & 1995.027 & 306.68 & 0.41 & 4 & 1.32 & 0.02 \\
\hline \multirow[t]{5}{*}{$06003-3102$} & HJ $3823 A C$ & $7.8-8.3$ & 1995.025 & 7.59 & 0.37 & 4 & 2.34 & 0.01 \\
\hline & & & 1995.027 & 7.71 & 0.27 & 5 & 2.29 & 0.01 \\
\hline & & & 1995.115 & 8.53 & 0.33 & 4 & 2.41 & 0.03 \\
\hline & & & ---- & ---- & ---- & -- & ---- & -- \\
\hline & & & 1995.056 & 7.93 & 0.52 & 15 & 2.35 & 0.05 \\
\hline $06047-4505$ & $H J 3834 A B$ & $5.9-9.4$ & 1995.071 & 218.11 & 0.55 & 4 & 5.72 & 0.04 \\
\hline \multirow[t]{4}{*}{$06048-4828$} & $D U N 23$ & $7.2-7.5$ & 1995.025 & 209.70 & 0.18 & 5 & 2.45 & 0.03 \\
\hline & & & 1995.027 & 208.96 & 0.16 & 6 & 2.50 & 0.02 \\
\hline & & & ---- & ---- & ---- & -- & ---- & -- \\
\hline & & & 1994.026 & 209.30 & 0.41 & 11 & 2.48 & 0.03 \\
\hline \multirow{6}{*}{$06344+1445$} & 5197 & $8.1-8.2$ & 1994.863 & 310.68 & 0.43 & 8 & 1.70 & 0.02 \\
\hline & & & 1995.071 & 309.20 & 1.45 & 5 & 1.69 & 0.02 \\
\hline & & & 1995.071 & 311.06 & 0.29 & 7 & 1.71 & 0.01 \\
\hline & & & 1995.112 & 309.07 & 1.07 & 5 & 1.65 & 0.02 \\
\hline & & & ---- & ---- & ---- & -- & ---- & -- \\
\hline & & & 1995.018 & 310.27 & 1.03 & 27 & 1.69 & 0.03 \\
\hline$(*)$ & 5197 & $8.1-8.2$ & 1995.100 & 309.70 & 0.80 & 2 & 1.82 & 0.10 \\
\hline \multirow[t]{6}{*}{$06546+1311$} & $5559 A B$ & $4.7-7.7$ & 1994.863 & 145.37 & 0.18 & 8 & 7.22 & 0.03 \\
\hline & & & 1995.025 & 144.63 & 0.37 & 6 & 7.06 & 0.08 \\
\hline & & & 1995.027 & 146.42 & 0.38 & 5 & 7.14 & 0.04 \\
\hline & & & 1995.115 & 144.72 & 0.19 & 6 & 7.11 & 0.02 \\
\hline & & & ---- & ---- & ---- & -- & ---- & -- \\
\hline & & & 1994.996 & 145.24 & 0.72 & 23 & 7.15 & 0.06 \\
\hline$(*)$ & $5559 A B$ & $4.7-7.7$ & 1995.100 & 145.40 & 0.40 & 2 & 7.12 & 0.10 \\
\hline \multirow[t]{5}{*}{$07201+2159$} & 5983 & $3.5-8.5$ & 1994.863 & 222.40 & 0.55 & 6 & 5.83 & 0.04 \\
\hline & & & 1995.025 & 224.03 & 0.37 & 5 & 5.65 & 0.07 \\
\hline & & & 1995.027 & 223.09 & 0.68 & 5 & 5.78 & 0.04 \\
\hline & & & ---- & ---- & ---- & -- & ---- & -- \\
\hline & & & 1994.949 & 223.18 & 0.87 & 15 & 5.80 & 0.05 \\
\hline$(*)$ & 5983 & $3.5-8.5$ & 1995.100 & 223.80 & 0.70 & 2 & 5.96 & 0.14 \\
\hline \multirow[t]{2}{*}{$07294-1500$} & $6126 A B$ & $6.1-7.7$ & 1994.863 & 21.53 & 0.49 & 9 & 1.86 & 0.05 \\
\hline & & & 1995.071 & 22.34 & 1.23 & 5 & 1.86 & 0.03 \\
\hline
\end{tabular}


Table 1. continued

\begin{tabular}{|c|c|c|c|c|c|c|c|c|}
\hline$W D S$ & ADS or NAME & magnitudes & $t$ & $\theta$ & $\sigma_{\theta}$ & $n_{\theta}^{0}$ & $\rho$ & $\sigma_{\rho}$ \\
\hline \multirow[t]{4}{*}{$07346+3153$} & $6175 A B$ & $2.0-2.9$ & 1995.027 & 71.39 & 0.29 & 6 & 3.44 & 0.04 \\
\hline & & & 1995.115 & 69.80 & 0.33 & 7 & 3.39 & 0.07 \\
\hline & & & ---- & ---- & ---- & -- & ---- & -- \\
\hline & & & 1995.074 & 70.53 & 0.85 & 12 & 3.42 & 0.05 \\
\hline \multirow[t]{4}{*}{$07346+3153$} & $6175 A B$ & $2.0-2.9$ & 1997.123 & 68.84 & 0.46 & 4 & 3.58 & 0.03 \\
\hline & & & 1997.123 & - & - & 0 & 3.73 & 0.07 \\
\hline & & & ---- & ---- & ---- & -- & ---- & -- \\
\hline & & & 1997.123 & 68.84 & 0.46 & 4 & 3.68 & 0.10 \\
\hline $07494-3033$ & $I 186$ & $8.2-8.4$ & 1995.071 & 245.77 & 2.33 & 5 & 1.45 & 0.02 \\
\hline (*)08024+0409 & 6532 & $8.5-10.4$ & 1995.100 & 275.60 & 0.90 & 2 & 1.45 & 0.05 \\
\hline \multirow[t]{6}{*}{$08095+3213$} & 6623 & $7.1-8.0$ & 1994.863 & 23.89 & 0.10 & 8 & 2.86 & 0.02 \\
\hline & & & 1995.025 & 24.28 & 0.21 & 5 & 2.90 & 0.03 \\
\hline & & & 1995.027 & 23.92 & 0.07 & 6 & 2.88 & 0.01 \\
\hline & & & 1995.115 & 23.05 & 0.77 & 5 & 2.75 & 0.03 \\
\hline & & & ---- & ---- & ---- & -- & ---- & -- \\
\hline & & & 1994.992 & 23.80 & 0.55 & 25 & 2.85 & 0.06 \\
\hline$(*)$ & 6623 & $7.1-8.0$ & 1995.100 & 23.80 & 1.00 & 2 & 2.95 & 0.15 \\
\hline \multirow[t]{5}{*}{$08122+1739$} & $6650 A B-C$ & $5.1-6.2$ & 1994.863 & 74.90 & 0.51 & 9 & 6.08 & 0.04 \\
\hline & & & 1995.025 & 74.75 & 0.06 & 5 & 6.08 & 0.01 \\
\hline & & & 1995.027 & 75.22 & 0.38 & 4 & 6.05 & 0.04 \\
\hline & & & ---- & ---- & ---- & -- & ---- & -- \\
\hline & & & 1994.949 & 74.93 & 0.44 & 17 & 6.07 & 0.04 \\
\hline$(*)$ & $6650 A B-C$ & $5.1-6.2$ & 1995.200 & 76.60 & 1.00 & 3 & 6.27 & 0.10 \\
\hline \multirow[t]{4}{*}{$08122+1739$} & $6650 A B-C$ & $5.1-6.2$ & 1997.123 & 73.12 & 0.30 & 5 & 5.89 & 0.06 \\
\hline & & & 1997.123 & 73.73 & 0.15 & 5 & 5.95 & 0.06 \\
\hline & & & ---- & ---- & ---- & -- & ---- & -- \\
\hline & & & 1997.123 & 73.43 & 0.38 & 10 & 5.92 & 0.06 \\
\hline $08221-4059$ & $H J 4087 A B$ & $7.7-8.0$ & 1995.071 & 266.54 & 1.48 & 4 & 1.45 & 0.01 \\
\hline \multirow[t]{6}{*}{$09144+5241$} & $7251 A B$ & $7.6-7.7$ & 1994.863 & 91.32 & 0.22 & 3 & 17.34 & 0.03 \\
\hline & & & 1995.025 & 91.27 & 0.29 & 6 & 17.34 & 0.06 \\
\hline & & & 1995.027 & 91.61 & 0.06 & 3 & 17.26 & 0.01 \\
\hline & & & 1995.134 & 91.40 & 0.15 & 4 & 17.36 & 0.05 \\
\hline & & & ---- & ---- & ---- & -- & ---- & -- \\
\hline & & & 1995.022 & 91.37 & 0.25 & 16 & 17.33 & 0.06 \\
\hline$(*)$ & $7251 A B$ & $7.6-7.7$ & 1995.100 & 91.80 & 0.30 & 2 & 17.39 & 0.13 \\
\hline \multirow[t]{2}{*}{$09144+5241$} & $7251 A B$ & $7.6-7.7$ & 1997.370 & 92.31 & 0.05 & 4 & 17.36 & 0.06 \\
\hline & & & 1997.370 & 92.64 & 0.15 & 4 & 17.32 & 0.02 \\
\hline
\end{tabular}


Table 1. continued

\begin{tabular}{|c|c|c|c|c|c|c|c|c|}
\hline$W D S$ & ADS or NAME & magnitudes & $t$ & $\theta$ & $\sigma_{\theta}$ & $n_{\theta}^{0}$ & $\rho$ & $\sigma_{\rho}$ \\
\hline \multirow[t]{7}{*}{$10200+1950$} & $7724 A B$ & $2.6-3.8$ & 1995.025 & 123.35 & 0.23 & 5 & 4.60 & 0.04 \\
\hline & & & 1995.027 & 124.81 & 0.17 & 6 & 4.61 & 0.02 \\
\hline & & & 1995.115 & 122.85 & 0.60 & 4 & 4.39 & 0.07 \\
\hline & & & 1995.132 & 124.16 & 0.13 & 5 & 4.60 & 0.01 \\
\hline & & & 1995.134 & 124.70 & 0.33 & 8 & 4.59 & 0.11 \\
\hline & & & ---- & ---- & ---- & -- & ---- & -- \\
\hline & & & 1995.089 & 124.26 & 0.63 & 24 & 4.59 & 0.07 \\
\hline$(*)$ & $7724 A B$ & $2.6-3.8$ & 1995.100 & 125.20 & 0.70 & 2 & 4.63 & 0.24 \\
\hline$(*) 11137+2008$ & 8094 & $7.6-7.8$ & 1995.100 & 324.00 & 1.90 & 2 & 0.62 & 0.07 \\
\hline (*)12306 + 0943 & 8575 & $8.1-8.4$ & 1995.700 & 243.40 & 0.60 & 2 & 1.39 & 0.14 \\
\hline \multirow[t]{4}{*}{$13491+2659$} & 9031 & $7.7-7.9$ & 1997.370 & 171.31 & 0.08 & 6 & 3.33 & 0.01 \\
\hline & & & 1997.370 & 170.78 & 0.11 & 6 & 3.36 & 0.01 \\
\hline & & & ---- & ---- & ---- & -- & ---- & -- \\
\hline & & & 1997.370 & 171.05 & 0.28 & 12 & 3.34 & 0.02 \\
\hline \multirow[t]{4}{*}{$13550-0804$} & $9053 A B$ & $6.5-7.7$ & 1995.132 & 97.87 & 0.48 & 6 & 3.57 & 0.05 \\
\hline & & & 1995.133 & 97.24 & 0.43 & 5 & 3.50 & 0.04 \\
\hline & & & ---- & ---- & ---- & -- & ---- & -- \\
\hline & & & 1995.132 & 97.70 & 0.45 & 10 & 3.53 & 0.04 \\
\hline (*)14587- 2739 & 9453 & $6.3-6.6$ & 1996.000 & 358.00 & 1.00 & 2 & 0.61 & 0.06 \\
\hline \multirow[t]{4}{*}{$15038+4739$} & 9494 & $5.3-6.2$ & 1997.660 & 54.53 & 0.77 & 5 & 1.99 & 0.01 \\
\hline & & & 1997.660 & 53.17 & 0.52 & 5 & 2.02 & 0.02 \\
\hline & & & ---- & ---- & ---- & -- & ---- & -- \\
\hline & & & 1997.660 & 53.85 & 0.94 & 10 & 2.00 & 0.02 \\
\hline \multirow[t]{4}{*}{$15183+2650$} & $9578 A a-B$ & $7.1-7.6$ & 1997.660 & 258.18 & 0.43 & 7 & 1.56 & 0.01 \\
\hline & & & 1997.660 & 258.75 & 0.25 & 7 & 1.54 & 0.01 \\
\hline & & & ---- & ---- & ---- & -- & ---- & -- \\
\hline & & & 1997.660 & 258.55 & 0.33 & 14 & 1.55 & 0.01 \\
\hline \multirow[t]{4}{*}{$15245+3721$} & $9626 B C$ & $7.2-7.8$ & 1997.660 & 10.60 & 0.39 & 5 & 2.25 & 0.01 \\
\hline & & & 1997.660 & 10.86 & 0.71 & 5 & 2.26 & 0.01 \\
\hline & & & ---- & ---- & ---- & -- & ---- & -- \\
\hline & & & 1997.660 & 10.73 & 0.59 & 10 & 2.26 & 0.01 \\
\hline
\end{tabular}


Table 1. continued

\begin{tabular}{|c|c|c|c|c|c|c|c|c|c|}
\hline$W D S$ & ADS or NAME & magnitudes & $t$ & $\theta$ & $\sigma_{\theta}$ & $n_{\theta}^{0}$ & $\rho$ & $\sigma_{\rho}$ & $n_{\rho}^{0}$ \\
\hline \multirow[t]{9}{*}{$15348+1032$} & $9701 A B$ & $4.2-5.2$ & 1994.430 & - & - & 0 & 3.99 & 0.00 & 1 \\
\hline & & & 1994.430 & 175.13 & 0.11 & 2 & 3.97 & 0.04 & 2 \\
\hline & & & 1994.430 & 175.13 & 0.11 & 1 & 3.91 & 0.00 & 1 \\
\hline & & & 1994.430 & 174.50 & 0.12 & 5 & 3.96 & 0.02 & 5 \\
\hline & & & 1995.195 & 175.05 & 1.52 & 5 & 3.66 & 0.10 & 5 \\
\hline & & & 1995.195 & 175.80 & 1.22 & 5 & 3.76 & 0.05 & 4 \\
\hline & & & 1995.460 & 174.09 & 0.34 & 7 & 4.02 & 0.02 & 7 \\
\hline & & & ---- & ---- & ---- & -- & ---- & -- & -- \\
\hline & & & 1994.994 & 174.37 & 0.49 & 24 & 3.90 & 0.13 & 25 \\
\hline$(*)$ & $9701 A B$ & $4.2-5.2$ & 1996.000 & 174.00 & 1.00 & 2 & 4.07 & 0.14 & 2 \\
\hline \multirow{6}{*}{$15559-0210$} & 9842 & $7.0-8.1$ & 1994.430 & - & - & 0 & 5.96 & 0.00 & 1 \\
\hline & & & 1994.430 & 350.99 & 0.20 & 5 & 5.98 & 0.05 & 5 \\
\hline & & & 1995.195 & 351.74 & 0.18 & 4 & 5.88 & 0.01 & 3 \\
\hline & & & 1995.460 & 351.31 & 0.37 & 8 & 6.00 & 0.03 & 8 \\
\hline & & & ---- & ---- & ---- & -- & ---- & -- & -- \\
\hline & & & 1995.050 & 351.32 & 0.40 & 17 & 5.97 & 0.06 & 17 \\
\hline$(*)$ & 9842 & $7.0-8.1$ & 1996.000 & 351.20 & 0.09 & 2 & 5.89 & 0.09 & 2 \\
\hline \multirow[t]{9}{*}{$16133+1332$} & $9969 A B$ & $7.5-7.7$ & 1994.430 & - & - & 0 & 4.09 & 0.00 & 1 \\
\hline & & & 1994.430 & 353.21 & 0.12 & 5 & 4.11 & 0.03 & 5 \\
\hline & & & 1994.515 & 353.21 & 0.12 & 1 & 4.34 & 0.00 & 1 \\
\hline & & & 1994.515 & 352.61 & 0.18 & 5 & 4.17 & 0.05 & 3 \\
\hline & & & 1994.515 & 352.69 & 0.27 & 7 & 4.06 & 0.04 & 7 \\
\hline & & & 1995.460 & 353.66 & 0.08 & 9 & 4.11 & 0.01 & 9 \\
\hline & & & 1995.499 & 353.87 & 0.26 & 6 & 4.11 & 0.01 & 6 \\
\hline & & & ---- & ---- & ---- & -- & ---- & -- & -- \\
\hline & & & 1994.949 & 353.29 & 0.51 & 30 & 4.10 & 0.03 & 32 \\
\hline$(*)$ & $9969 A B$ & $7.5-7.7$ & 1995.500 & 352.60 & 0.90 & 2 & 4.05 & 0.07 & 2 \\
\hline \multirow[t]{6}{*}{$16160+0721$} & 9982 & $9.3-9.8$ & 1997.660 & 20.02 & 0.52 & 5 & 3.21 & 0.03 & 5 \\
\hline & & & 1997.660 & 20.28 & 0.17 & 5 & 3.21 & 0.01 & 5 \\
\hline & & & 1997.666 & 19.84 & 0.50 & 5 & 3.21 & 0.03 & 5 \\
\hline & & & 1997.666 & 20.43 & 1.02 & 4 & 3.21 & 0.03 & 5 \\
\hline & & & ---- & ---- & ---- & -- & ---- & -- & -- \\
\hline & & & 1997.663 & 20.02 & 0.49 & 18 & 3.20 & 0.04 & 20 \\
\hline \multirow[t]{4}{*}{$16289+1825$} & $10075 A B$ & $7.8-7.8$ & 1997.663 & 125.19 & 0.32 & 6 & 1.96 & 0.02 & 5 \\
\hline & & & 1997.663 & 126.07 & 0.13 & 5 & 1.95 & 0.01 & 5 \\
\hline & & & ---- & ---- & ---- & -- & ---- & -- & -- \\
\hline & & & 1997.663 & 125.69 & 0.41 & 10 & 1.96 & 0.02 & 11 \\
\hline
\end{tabular}


Table 1. continued

\begin{tabular}{|c|c|c|c|c|c|c|c|c|}
\hline$W D S$ & ADS or NAME & magnitudes & $t$ & $\theta$ & $\sigma_{\theta}$ & $n^{0}$ & $\rho$ & $\sigma_{\rho}$ \\
\hline$(*) 16294-2626$ & 10074 & $1.0-5.4$ & 1996.100 & 277.00 & 1.30 & 4 & 2.77 & 0.15 \\
\hline \multirow[t]{7}{*}{$17153-2636$} & $10417 A B$ & $5.3-5.3$ & 1994.430 & - & - & 0 & 4.68 & 0.00 \\
\hline & & & 1994.430 & 148.92 & 0.17 & 5 & 4.74 & 0.04 \\
\hline & & & 1995.460 & 148.49 & 0.12 & 9 & 4.77 & 0.01 \\
\hline & & & 1995.499 & 148.34 & 0.26 & 7 & 4.77 & 0.06 \\
\hline & & & 1995.499 & 148.19 & 0.30 & 6 & 4.80 & 0.09 \\
\hline & & & ---- & ---- & ---- & -- & ---- & -- \\
\hline & & & 1995.266 & 148.46 & 0.33 & 29 & 4.77 & 0.06 \\
\hline$(*)$ & $10417 A B$ & $5.3-5.3$ & 1996.100 & 147.80 & 0.70 & 4 & 4.73 & 0.07 \\
\hline \multirow[t]{7}{*}{$17146+1423$} & $10418 A B$ & $3.5-5.4$ & 1994.430 & - & - & 0 & 4.32 & 0.00 \\
\hline & & & 1994.430 & 105.58 & 0.43 & 6 & 4.71 & 0.04 \\
\hline & & & 1994.515 & 105.58 & 0.43 & 1 & 4.74 & 0.00 \\
\hline & & & 1994.515 & 105.72 & 0.34 & 5 & 4.63 & 0.07 \\
\hline & & & 1995.460 & 107.82 & 0.37 & 8 & 4.94 & 0.04 \\
\hline & & & ---- & ---- & ---- & -- & ---- & -- \\
\hline & & & 1994.863 & 106.56 & 1.14 & 18 & 4.81 & 0.13 \\
\hline$(*)$ & $10418 A B$ & $3.5-5.4$ & 1996.100 & 105.00 & 1.40 & 4 & 4.70 & 0.18 \\
\hline$(*) 18101+1629$ & 11123 & $6.5-7.3$ & 1996.100 & 219.80 & 1.00 & 3 & 1.17 & 0.05 \\
\hline $18154+1946$ & 11208 & $8.2-9.0$ & 1997.666 & 30.38 & 0.43 & 5 & 5.19 & 0.05 \\
\hline \multirow[t]{7}{*}{$18443+3940$} & $11635 A B$ & $5.1-6.0$ & 1994.430 & - & - & 0 & 2.47 & 0.00 \\
\hline & & & 1994.430 & 350.95 & 1.58 & 6 & 2.41 & 0.04 \\
\hline & & & 1995.460 & 351.72 & 0.19 & 10 & 2.47 & 0.01 \\
\hline & & & 1995.460 & 353.10 & 1.00 & 8 & 2.53 & 0.04 \\
\hline & & & 1995.499 & 353.23 & 1.18 & 9 & 2.51 & 0.05 \\
\hline & & & ---- & ---- & ---- & -- & ---- & -- \\
\hline & & & 1991.235 & 352.46 & 1.18 & 35 & 2.48 & 0.05 \\
\hline$(*)$ & $11635 A B$ & $5.1-6.0$ & 1996.100 & 351.00 & 0.97 & 3 & 2.46 & 0.09 \\
\hline \multirow[t]{6}{*}{$18443+3940$} & $11635 C c-D$ & $5.1-5.4$ & 1994.430 & 83.21 & 0.83 & 1 & 2.35 & 0.00 \\
\hline & & & 1994.430 & 86.72 & 0.27 & 6 & 2.34 & 0.04 \\
\hline & & & 1995.499 & 84.05 & 0.41 & 8 & 2.30 & 0.04 \\
\hline & & & 1995.499 & 83.21 & 0.83 & 8 & 2.28 & 0.04 \\
\hline & & & ---- & ---- & ---- & -- & ---- & -- \\
\hline & & & 1995.227 & 84.48 & 1.53 & 26 & 2.31 & 0.05 \\
\hline$(*)$ & $11635 C c-D$ & $5.1-5.4$ & 1996.100 & 84.40 & 0.70 & 3 & 2.31 & 0.10 \\
\hline
\end{tabular}


Table 1. continued

\begin{tabular}{|c|c|c|c|c|c|c|c|c|}
\hline$W D S$ & ADS or NAME & magnitudes & $t$ & $\theta$ & $\sigma_{\theta}$ & $n^{0}$ & $\rho$ & $\sigma_{\rho}$ \\
\hline \multirow[t]{6}{*}{$19143+1904$} & 12201 & $7.9-9.4$ & 1994.430 & - & - & 0 & 2.36 & 0.00 \\
\hline & & & 1994.430 & 235.72 & 2.94 & 4 & 2.35 & 0.08 \\
\hline & & & 1995.460 & 238.33 & 0.23 & 8 & 2.18 & 0.03 \\
\hline & & & 1995.460 & 238.19 & 0.26 & 9 & 2.13 & 0.04 \\
\hline & & & ---- & ---- & ---- & -- & ---- & -- \\
\hline & & & 1995.264 & 238.13 & 0.40 & 21 & 2.19 & 0.09 \\
\hline$(*)$ & 12201 & $7.9-9.4$ & 1995.600 & 238.00 & 1.00 & 2 & 2.17 & 0.05 \\
\hline (*)20012-3835 & HDO 294 & $8.2-8.9$ & 1995.600 & 24.20 & 0.60 & 2 & 1.12 & 0.10 \\
\hline$(*) 20462+1554$ & 14270 & $7.6-8.4$ & 1995.600 & 9.60 & 0.70 & 2 & 5.98 & 0.14 \\
\hline (*)21031 + 0132 & $14573 A B$ & $6.7-7.3$ & 1995.600 & 119.50 & 1.20 & 2 & 1.30 & 0.10 \\
\hline \multirow[t]{6}{*}{$22038+6438$} & $15600 A a-B$ & $4.6-6.5$ & 1994.460 & 275.33 & 0.51 & 4 & 7.92 & 0.06 \\
\hline & & & 1994.460 & 276.27 & 0.22 & 5 & 7.86 & 0.05 \\
\hline & & & 1995.499 & 275.85 & 0.55 & 7 & 7.92 & 0.09 \\
\hline & & & 1995.499 & 275.56 & 0.39 & 5 & 7.84 & 0.06 \\
\hline & & & ---- & ---- & ---- & -- & ---- & -- \\
\hline & & & 1995.054 & 275.78 & 0.56 & 21 & 7.89 & 0.08 \\
\hline$(*)$ & $15600 A a-B$ & $4.6-6.5$ & 1995.600 & 276.80 & 1.00 & 2 & 7.89 & 0.10 \\
\hline
\end{tabular}

Acknowledgements. This work was financed by the research project XUGA 24301B96 directed by J.A. Docobo and supported by Xunta de Galicia (Spain).

\section{References}

Abad C., Della Prugna F., 1995, A\&AS 111, 229

Aitken R.G., 1932, New General Catalogue of Double Stars (ADS). Carnegie Institution of Washington No. 417
Brosche P., Sinachopoulos D., 1989, Bull. Inform. CDS, 36 Prieto C., 1997, A\&AS 121, 405

Valbousquet A., 1980, A\&AS 40, 347

Worley C.E., Douglas G.G., 1996, The Washington Visual Double Star Catalogue, 1996.0 Electronic version Naval Observatory WWW (http://aries.usno.navy.mil/ad/wds/.) 